\title{
Echokardiografische Untersuchung klinisch gesunder Frettchen ohne Sedation
}

\section{Echocardiographic measurements in clinically healthy ferrets without sedation}

Autoren

Nicolai Hildebrandt, Estelle Henrich, Matthias Schneider

Institut

Klinik für Kleintiere, Innere Medizin, Klinikum

Veterinärmedizin, Justus-Liebig-Universität Gießen

Schlüsselwörter

Herzerkrankung, Herzultraschall, B-Mode, M-Mode, linker Vorhof

Key words

Cardiac disease, echocardiography, B-mode, M-mode, left atrium

eingereicht 17.10 .2020

akzeptiert $\quad 30.12 .2020$

Bibliografie

Tierarztl Prax Ausg K Kleintiere Heimtiere 2021; 49: 104-109

DOI 10.1055/a-1380-7464

ISSN 1434-1239

(c) 2021. Thieme. All rights reserved.

Georg Thieme Verlag KG, Rüdigerstraße 14,

70469 Stuttgart, Germany

Korrespondenzadresse

Nicolai Hildebrandt

Klinik für Kleintiere, Innere Medizin

Klinikum Veterinärmedizin

Justus-Liebig-Universität Gießen

Frankfurter Straße 114

35392 Gießen

Deutschland

Nicolai.B.Hildebrandt@vetmed.uni-giessen.de

\section{ZUSAMMENFASSUNG}

Gegenstand und Ziel Herzerkrankungen, in erster Linie Klappeninsuffizienzen und Kardiomyopathien, spielen auch beim Frettchen eine wichtige Rolle. Ziel dieser Studie war, am wachen gesunden Frettchen gewichtsbezogene Referenzintervalle für echokardiografische Routineparameter im B- und M-Mode zu etablieren, insbesondere des linken Vorhofs.

Material und Methoden Bei 18 gesunden, unsedierten Frettchen erfolgte eine echokardiografische Untersuchung in rechter Seitenlage. In 3 M-Mode-Schnitten (linker Ventrikel, Mitralklappenspitze, Aortenwurzel) aus der rechtsparasternalen

Längsachse wurden 3 Herzzyklen mit guter Wandbegrenzung ausgewertet und der Mittelwert ermittelt. Im zweidimensionalen B-Mode-Bild wurden aus dem rechtsparasternalen Kurzachsenblick auf Höhe der Aortenklappe der linke Vorhof (LA) sowie die Aorta (Ao) gemessen und der Quotient LA/Ao errechnet. Ergebnisse Die 9 männlichen und 9 weiblichen Frettchen (jeweils kastriert) hatten ein mittleres Alter von 2,6 \pm 1,3 Jahren und ein mittleres Gewicht von 1,04 00,31 kg. Die M-Mode-Messungen auf Ventrikelebene bzw. Aortenwurzel ließen sich bei allen Tieren durchführen. Es zeigten sich signifikante Effekte des Körpergewichts auf den Durchmesser des Ventrikels in Diastole und Systole sowie Aorta und Vorhof. Die Messung im B-Bild war oftmals nur an einem Standbild ausreichend möglich. Auch hier ergab sich eine signifikante Abhängigkeit von Ao und LA vom Gewicht. Errechnete Parameter wie die Verkürzungsfraktion und der Quotient LA/Ao waren unabhängig vom Gewicht.

Schlussfolgerung und klinische Relevanz Die Echokardiografie im B- und M-Mode stellt am wachen Frettchen eine praxistaugliche Untersuchungstechnik zur Ermittlung von Standardparametern für die morphologische Beurteilung des Herzens dar. Das Körpergewicht muss hierbei jeweils berücksichtigt werden.

\section{ABSTRACT}

Objective Cardiac diseases, predominantly acquired diseases such as valvular insufficiency and cardiomyopathies, are a common problem in domestic ferrets. Aim of the present study was to establish body-weight related echocardiographic reference intervals for standard B- and M-mode parameters, especially of the left atrium, in clinically healthy ferrets without sedation.

Material and methods Eighteen client-owned healthy, nonsedated ferrets underwent echocardiography in right lateral recumbency. Mean values were calculated from the evaluation of 3 cardiac cycles with good wall differentiation within $3 \mathrm{M}$-mode measurements in the right parasternal long axis view (level of the chordae tendinae, tip of the mitral valve and level of the aortic root and left atrium). Additionally, measurements of the left atrium (LA) and aorta (Ao) were performed in the short axis view at the level of the aorta and the LA/Ao ratio was calculated. Results The 9 neutered male and 9 neutered female ferrets had a mean age of $2.6 \pm 1.3$ years and a mean body weight of $1.04 \pm 0.31 \mathrm{~kg}$. M-mode measurements of the left ventricle and 
the aortic root were possible in all animals. Body weight exerted a significant effect on the ventricular dimensions in diastole and systole as well as on the measurements of the left atrium and the aorta. In the B-mode evaluations, measurements of the aorta and the left atrium were frequently only performable on a static image. A significant effect of body weight on the dimensions of both aorta and left atrium were also apparent in the B-mode measurements. Calculated values such as fractional shorting and LA/Ao-ratio proved to be independent of body weight.

Conclusion and clinical relevance Standard B- and M- parameters may be used in routine examinations in order to evaluate cardiac morphology in the non-sedated ferret. Consideration of the body weight is warranted in this species.

\section{Einleitung}

Beim Frettchen ist ähnlich wie bei anderen Tierarten inzwischen eine Vielzahl an angeborenen [1][2][3] wie auch erworbenen [3] [4][5] kardiovaskulären Abnormalitäten beschrieben, wobei wie bei Hund und Katze erworbene Herzerkrankungen dominieren [3][4] [5][6]. In erster Linie werden beim Frettchen Klappeninsuffizienzen beschrieben, die im Unterschied zu anderen Kleintieren am häufigsten die Aortenklappe und erst an zweiter Stelle die Mitralklappe betreffen [6]. Seltener sind primär myokardiale Erkrankungen wie eine nicht näher abzuklärende linksventrikuläre Hypertrophie oder eine dilatative Kardiomyopathie (DCM) dokumentiert [3][4][5][6]. Hinzu kommt ein Anteil mit nicht näher differenzierter Ventrikeldilatation [6]. Die Infektion mit Dirofilaria immitis stellt ebenfalls eine kardiale Erkrankung beim Frettchen dar [3][7]. Bei verzeichneten Herzrhythmusstörungen handelt es sich überwiegend AV-Blöcke [3][6][8]. Das Spektrum an dokumentierten angeborenen Anomalien beim Frettchen umfasst v. a. Shuntverbindungen wie den Atrium- und Ventrikelseptumdefekt, aber auch Kombinationsmissbildungen wie die Fallot'sche Tetralogie [1][2][3].

Wie in anderen Bereichen der Kleintiermedizin ist auch beim Frettchen der Herzultraschall inzwischen wesentlicher Baustein der kardialen Diagnostik. Es können spezifische Diagnosen gestellt werden und somit die unspezifischeren Befunde (z. B. die in älteren Beschreibungen oft verwendete „congestive heart failure“) ersetzt werden [6][9]. Zudem lässt sich der Schweregrad der jeweiligen Erkrankung bestimmen. Referenzwerte für die Echokardiografie beim Frettchen wurden aufgrund der ausgeprägten Unruhe bzw. Beweglichkeit der Tiere bisher primär in Sedation bzw. Narkose erhoben [10][11]. Lediglich in einer Arbeit konnten Richtwerte für unsedierte Tiere ermittelt werden [12]. Am wachen Frettchen erwies es sich allerdings als schwierig, die linksatriale Dimension zu ermitteln [12]. Eine Erfassung der Größe des linken Vorhofes aus dem zweidimensionalen Querschnitt auf Höhe der Herzbasis, wie sie bei Hund und Katze aktueller Standard ist [13][14][15], wurde außerdem bisher weder am sedierten/narkotisierten noch am wachen Tier etabliert [10][11][12]. Frettchen zeigen physiologischerweise eine sehr große Gewichtsspanne. Im Vergleich können schwerere Tiere das 3- bis 4-fache Körpergewicht von leichten Tieren aufweisen [10][12]. Trotz dieser Tatsache konnte bisher keine signifikante Korrelation von echokardiografischen Messwerten zum Körpergewicht gezeigt werden. Ausschließlich die jüngste Arbeit ergab höhere Messwerte bei den durchschnittlich schwereren männlichen Tieren [12].

Ziel dieser Arbeit war, die Möglichkeit einer echokardiografischen Untersuchung am unsedierten Frettchen zu prüfen und gewichtsbezogene Referenzintervalle zu etablieren. Ferner sollte un- tersucht werden, ob die Messung des linken Vorhofs im B-Bild aus der Kurzachse möglich ist.

\section{Material und Methoden}

\section{Patienten}

Im Zeitraum von Mai 2002 bis Februar 2007 wurden prospektiv klinisch gesunde Frettchen in die Studie einbezogen. Der Grund der Vorstellung war eine von den Besitzern gewünschte Gesundheitskontrolle. Als gesund wurden Tiere bewertet, die anamnestisch und in der klinischen Untersuchung keine Abweichungen zeigten. Weiteres Inklusionskriterium war ein Alter von mindestens 6 Monaten.

\section{Echokardiografische Untersuchung}

Die echokardiografische Untersuchung erfolgte am und sedierten Tier in rechter Seitenlage unter Verwendung eines Ultraschallsystems mit einem 7,0-MHz-Sektorschallkopf und paralleler EKG-Aufzeichnung durchgeführt (Toshiba Powervision oder Aplio, Neuss, D). Die Tiere wurden durch Angebot einer schmackhaften Vitaminpaste (Gimpet-Paste ${ }^{\circledR}$ oder Nutrical ${ }^{\circledR}$ ) bzw. falls notwendig mithilfe eines Nackengriffs in der gewünschten Seitenlage gehalten ( $>$ Abb. 1). Die Untersuchung beinhaltete standardmäßige echokardiografische Anschallungspositionen, wie sie für Hund und Katze beschrieben sind [16]. Aus dem rechtsparasternalen 4-Kammer-Längsachsenblick erfolgten 2 M-Mode-Aufzeichnungen auf Höhe der Sehnenfäden bzw. der Mitralklappenspitze. Aus der rechtsparasternalen Längsachse mit Blick in den linksventrikulären Ausflusstrakt wurde die M-Mode-Messung senkrecht durch die Aortenwurzel durchgeführt. Jeweils ein M-Mode-Bild mit einem Zeitfenster von 2,5 Sekunden wurde gespeichert. In einem rechtsparasternalen Kurzachsenblick auf Höhe der Aortenklappe erfolgte die Aufzeichnung der Aorta und des linken Vorhofs im zweidimensionalen Bild ( $\mathbf{A b b}$. 2). Hierfür wurde ein Loop mit 5 Herzzyklen aufgezeichnet.

\section{Durchführung der Messungen}

Alle Messungen wurden offline mit einer Software (MicroDicom ${ }^{\circledR}$, Sofia, Bulgarien) vorgenommen.

Messungen im M-Mode Die Messungen erfolgten an den Bildern nach humanmedizinisch definierten Kriterien [17]. Bei allen Dimensionsbestimmungen kam die „Leading-edge“-Messmethode zum Einsatz. Die Messungen erfolgten 3-fach, an 3 Komplexen mit der besten Wandabgrenzung und nicht zwingend konsekutiv.

Auf Ebene der Sehnenfäden wurden die Dimensionen des interventrikulären Septums, der linken Kammer und der linksven- 

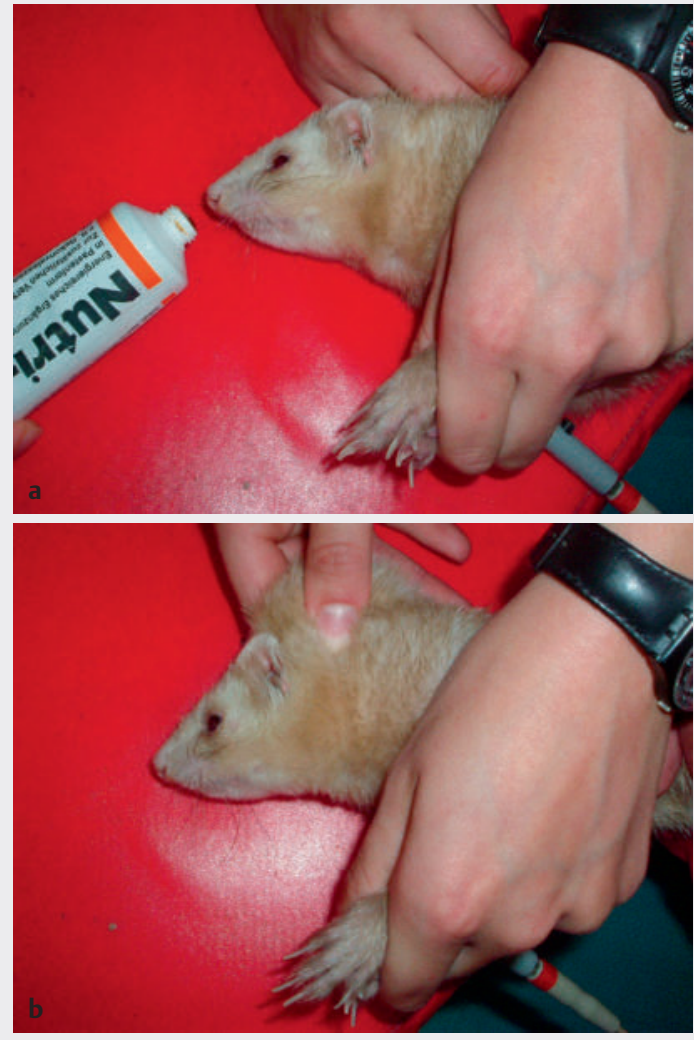

- Abb. 1 Echokardiografische Untersuchung eines Frettchens in rechter Seitenlage mit Angebot einer schmackhaften Vitaminpaste (a) oder Fixation durch Nackengriff (b). Quelle: $\odot$ N. Hildebrandt.

- Fig. 1 Echocardiography of a ferret in right lateral recumbency either by feeding tasty vitamin paste (a) or by restraining the patient in that position with a neck grip (b). Source: @ N. Hildebrandt.

trikulären freien Wand sowohl in der Diastole (IVSd, LVDd, LVWd), definiert anhand des EKGs (Beginn QRS-Komplex), als auch in der Systole (IVSs, LVDs, LVWs) zum Zeitpunkt des kleinsten linksventrikulären Durchmessers erfasst. Aus dem Unterschied zwischen diastolischem und systolischem Durchmesser der linken Hauptkammer wurde die Verkürzungsfraktion („fractional shortening“; FS) errechnet und aus dem zugehörigen R-R-Abstand im EKG die aktuelle Herzfrequenz ermittelt. An der Mitralklappe erfolgte die Bestimmung der EPSS („E-point septal separation“) als Abstand zwischen Mitralklappenspitze und interventrikulärem Septum in der ersten diastolischen Öffnungsphase der Mitralklappe sowie der maximalen Höhe der Mitralklappenöffnung (DE-Amplitude). Auf Höhe der Aortenwurzel wurde der Durchmesser der Aorta in der Diastole (Beginn des QRS-Komplexes) und des linken Vorhofs zum Zeitpunkt der höchsten Aortenwandbewegung gemessen.

Messungen im B-Mode Die Messungen von Aorta und linkem Vorhof erfolgten in Anlehnung an die beim Hund beschriebene Vorgehensweise [14]. Die Messpunkte wurden mittels „Inner-edge“Messung in der frühen Diastole nach Schluss der Aortenklappen gesetzt. Nach Möglichkeiten wurden 3 der 5 aufgezeichneten Herzzyklen mit ausreichender Abgrenzung ausgewertet.

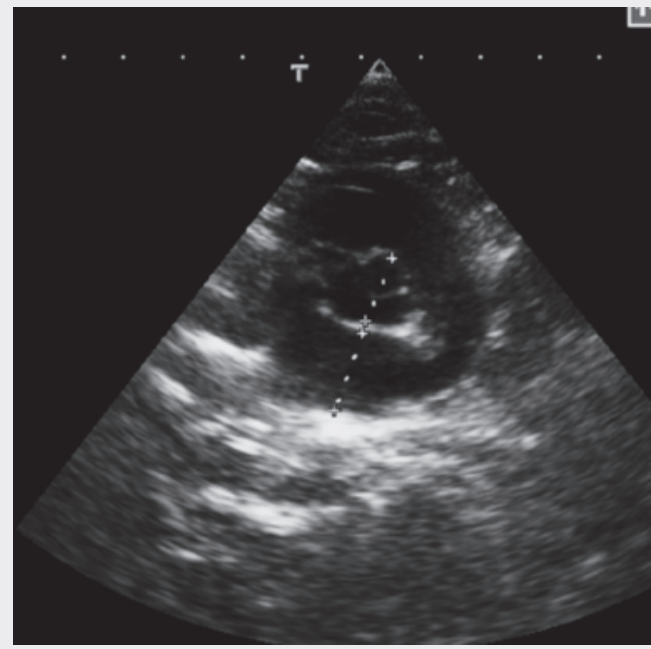

- Abb. 2 Erfassung der Dimension des linken Vorhofs (LA) im Verhältnis zur Aorta (Ao) als LA/Ao aus dem zweidimensionalen rechtsparasternalen Kurzachsenblick in Anlehnung an die Messung nach Hansson et al. [14] beim Hund. Die Dimensionen werden in der frühen Diastole bei geschlossener Aortenklappe als "Inner-edge“Messung erfasst. Quelle: @ N. Hildebrandt.

-Fig. 2 Estimation of the left atrial (LA) dimension in relation to the aorta (Ao) as LA/Ao from the two-dimensional right parasternal short axis view adopted to the quantification of the left atrium by Hansson et al. [14] in dogs. The measurements are performed in diastole when the aortic valves are closed as an inner edge method. Source: @ N. Hildebrandt.

Aus den oben beschriebenen Messwerten wurden folgende Quotienten errechnet:

- FS des linken Ventrikels

- Verhältnis linker Vorhof zu Aorta im M-Mode: LA/Ao-M

- Verhältnis linker Vorhof zu Aorta im B-Mode: LA/Ao-B

\section{Statistische Auswertung}

Die statistische Auswertung erfolgte unter Zuhilfenahme einer kommerziellen Software (GraphPadPrism 6). Die Daten wurden auf ihre Normalverteilung (D‘Agostino \& Pearson omnibus) geprüft und entsprechend als Mittelwert \pm Standardabweichung bzw. als Median und Range angegeben. Der Zusammenhang zwischen Körpergewicht und Größendimension wurde mittels linearer Regressionsanalyse geprüft. Zur Ermittlung von Unterschieden zwischen männlichen und weiblichen Tieren diente ein ungepaarter t-Test. Die unterschiedlichen Messmethoden in Bezug auf das linke Atrium (Verhältnis aus linkem Vorhof und Aorta) aus M-Mode (Längsachse des linksventrikulären Ausflusstrakts) und B-Mode (rechtsparasternale Kurzachse auf Höhe der Aorta) wurden über einen ungepaarten $\mathrm{t}$-Test verglichen. Ein $\mathrm{p}$-Wert $<0,05$ galt als statistisch signifikant.

\section{Ergebnisse}

Alle 18 Frettchen tolerierten die Untersuchung in rechter Seitenlage durch Angebot einer schmackhaften Vitaminpaste bzw. unter Fi- 


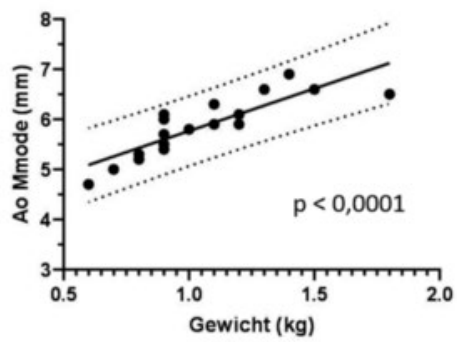

- Abb. 3 Darstellung der Gewichtsverteilung männlicher und weiblicher Tiere. Der Unterschied zwischen beiden Geschlechtern war signifikant $(p=0,0020)$. Quelle: $\odot N$. Hildebrandt.

- Fig. 3 Results of body weight distribution between female and male ferrets. A significant difference between both sexes was found $(p=0.0020)$. Source: $\odot$ N. Hildebrandt.

xierung mittels Nackengriff. Es handelte sich um 9 männliche und 9 weibliche Tiere (jeweils kastriert) im Alter von 2,6 \pm 1,3 Jahren. Das mittlere Körpergewicht betrug 1,04 $\pm 0,31 \mathrm{~kg}$ (Bereich 0,6-1,8 kg), wobei männliche Frettchen signifikant $(p=0,0020)$ schwerer waren als weibliche $(1,23 \pm 0,31 \mathrm{~kg}$ vs. $0,86 \pm 0,15 \mathrm{~kg})$ ( Abb. 3).

Die Herzfrequenz der Tiere lag während der Untersuchung bei 251,8 \pm 31,3 Schlägen/Minute. Die M-Mode-Aufzeichnung und dreifache Ausmessung auf Ventrikelebene und Aortenwurzel war immer möglich. Eine ausreichende M-Mode-Aufzeichnung der Mitralklappe gelang bei 15/18 Tieren. Die Aufzeichnung von 5 Herzzyklen im B-Mode ermöglichte bei den meisten Tieren (13/18) nur eine einmalige Ausmessung bei ausreichender Bildqualität. Die Messdaten sind in > Tab. 1 dargestellt. Mit Ausnahme von FS und LVDs wiesen die Werte aller Parameter Normalverteilung auf.

Die Ventrikeldimensionen in Diastole $(11,7 \pm 1,6 \mathrm{~mm})$ und Systole $(8,0 \pm 1,7 \mathrm{~mm})$ waren signifikant $(p<0,0001 ; p=0,0003)$ mit dem Gewicht korreliert. Von den Wandstärken des linken Ventrikels zeigte lediglich das interventrikuläre Septum in der Diastole eine Korrelation zum Körpergewicht ( $p=0,0338$ ). Die freie linksventrikuläre Wand in der Diastole $(3,4 \pm 0,3 \mathrm{~mm})$ wies keine signifikante Korrelation mit dem Gewicht auf $(p=0,1013)$. Die absoluten Dimensionen von Vorhof und Aorta im M-Mode (7,2 $\pm 1,3$ bzw. 5,9 $\pm 0,6 \mathrm{~mm}$ ) und B-Mode (6,7 $\pm 1,2 \mathrm{~mm}$ bzw. 5,3 $\pm 0,6 \mathrm{~mm}$ ) waren signifikant mit dem Gewicht korreliert (LA im M-Mode $\mathrm{p}=0,0077$; Ao im M-Mode $<0,0001$; LA im B-Mode <0,0001; Ao im B-Mode <0,0001), nicht aber das Verhältnis LA/Ao (M-Mode 1,2 $\pm 0,1 ; p=0,5914$ und B-Mode 1,3 $\pm 0,1 ; p=0,0586)$ und die FS $(32,4 \pm 6,9 \% ; p=0,4873)$. Die Messwerte der genannten Parameter in Relation zum Körpergewicht der 18 Frettchen sind in $>$ Abb. 4 dargestellt.

\section{Diskussion}

Die echokardiografische Untersuchung am nicht sedierten Frettchen konnte in der vorliegenden Untersuchung bei allen 18 Tieren erfolgreich vorgenommen werden. Dies deckt sich mit den Resultaten einer anderen Arbeit [12], in der sich eine Erfolgsquote von $96 \%$ ergab. Eine Sedation oder Narkose der Tiere zur Durchführung dieser Diagnostik ist daher entgegen Empfehlungen in der Literatur [10][11] nicht zwingend erforderlich.
- Tab. 1 Ergebnisse der echokardiografischen Messungen (B- und M-Mode-Verfahren) für 18 Frettchen, dargestellt als Mittelwert \pm Standardabweichung bzw. als Median und Range.

- Table 1 Results of the echocardiographic measurements (B- and $\mathrm{M}$-mode) for 18 ferrets. Results are provided as mean \pm standard deviation and as median and range, respectively.

\begin{tabular}{|c|c|}
\hline Parameter & Messwert \\
\hline Herzfrequenz (Schläge/min) & $251,8 \pm 31,3$ \\
\hline IVSd (mm) & $3,0 \pm 0,3$ \\
\hline IVSs (mm) & $3,0 \pm 0,4$ \\
\hline LVDd (mm) & $11,7 \pm 1,6$ \\
\hline LVDs (mm) & $8,0(5,9-10,6)$ \\
\hline LVWd (mm) & $3,4 \pm 0,3$ \\
\hline LVWs (mm) & $5,6 \pm 0,5$ \\
\hline FS (\%) & $29,5(22-43)$ \\
\hline LA aus M-Mode (mm) & $7,2 \pm 1,3$ \\
\hline AO aus M-Mode (mm) & $5,9 \pm 0,6$ \\
\hline LA/AO aus M-Mode & $1,2 \pm 0,1$ \\
\hline EPSS (mm) & $1,2 \pm 0,6$ \\
\hline DE-Amp (mm) & $3,6 \pm 0,9$ \\
\hline LA aus B-Mode (mm) & $6,7 \pm 1,2$ \\
\hline AO aus B-Mode (mm) & $5,3 \pm 0,6$ \\
\hline LA/AO aus B-Mode & $1,3 \pm 0,1$ \\
\hline \multicolumn{2}{|c|}{$\begin{array}{l}\text { IVSd/IVSs = interventrikuläres Septum in der Diastole bzw. Systole; } \\
\text { LVDd/LVDs = linksventrikulärer Durchmesser in der Diastole bzw. } \\
\text { Systole; LVWd/LVWs = linksventrikuläre Wand in der Diastole bzw. } \\
\text { Systole; FS = Verkürzungsfraktion („fractional shortening); LA= linker } \\
\text { Vorhof; Ao = Aorta; LA/Ao= Quotient aus linkem Vorhof und Aorta; } \\
\text { EPSS= „E-point septal separation“; DE-Amp = DE-Amplitude }\end{array}$} \\
\hline
\end{tabular}

Die Dreifachmessung im M-Mode auf Ventrikel- und Aortenebene gelang immer, allerdings nicht unbedingt an 3 aufeinanderfolgenden Komplexen, da es durch kleine Bewegungen der Tiere immer wieder zu einer suboptimalen Anschallung kam (>Abb.5). Der Effekt der Abwehrbewegung auf die Messposition bei Frettchen ist in vergleichbarer Weise für die Doppler-Untersuchung beschrieben [12]. Diese Probleme durch Bewegungen waren auch der Grund, weshalb die Auswertung der Mitralklappenbewegung im M-Mode gar nicht und die Ausmessung von Vorhof und Aorta im B-Mode nur als einfache Messung erfolgen konnte. Die aktuelle Studie zeigt zum ersten Mal für das Frettchen eine Abhängigkeit der Dimension echokardiografischer Parameter vom Körpergewicht, wie sie beim Hund [18] und bei der Katze [19] bereits bekannt ist. In einer früheren Arbeit [11] am narkotisierten Frettchen war dies nicht nachweisbar, hatten die Frettchen dieser Studie mit 0,5-1,2 kg (Median $0,7 \mathrm{~kg}$ ) ein deutlich geringeres Körpergewicht und eine niedrigere Gewichtsspanne als die Tiere der vorliegenden Studie (Median $0,9 \mathrm{~kg}$, Range 0,6-1,8 kg). Als hinweisend für den Einfluss des Gewichts auf die sonografisch ermittelten Größendimensionen beim Frettchen zeigte eine Untersuchung [12] einen signifikanten Unter- 

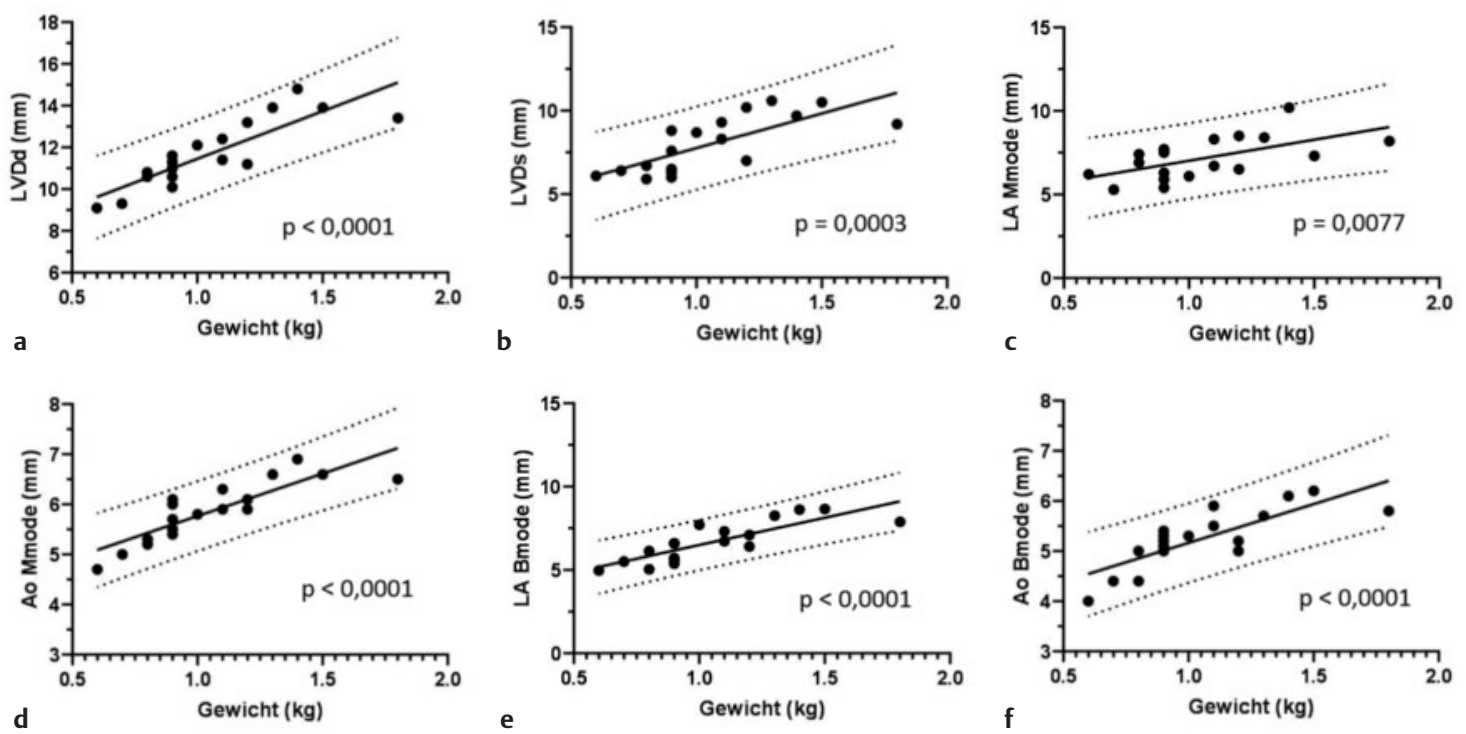

- Abb.4 Korrelation (lineare Regressionsanalyse) der Parameter LVWd und LVWs (linksventrikuläre Wand in der Diastole bzw. Systole) sowie LA (linker Vorhof) und Ao (Aorta) im M- und B-Mode mit dem Körpergewicht der untersuchten Frettchen. In allen Fällen ergab sich eine signifikante Korrelation: LVWd und LVWs (a, b): 11,7 $\pm 1,6 \mathrm{~mm}, \mathrm{p}<0,0001$ bzw. 8,0 $\pm 1,7 \mathrm{~mm}, \mathrm{p}=0,0003$; LA (c) und Aorta (d) im M-Mode: 7,2 $\pm 1,3, \mathrm{p}=0,0077$ bzw. 5,9 $\pm 0,6 \mathrm{~mm}, \mathrm{p}<0,0001$; LA (e) und Aorta (f) im B-Mode: 6,7 $\pm 1,2 \mathrm{~mm}$ bzw. 5,3 $\pm 0,6 \mathrm{~mm}$, jeweils $p<0,0001$. Quelle: ๑ N. Hildebrandt.

-Fig. 4 Linear regression analysis of body weight and the B- and M-mode parameters LVWd and LVWs (left ventricular internal diameter in diastole and systole, respectively), LA (left atrium) and Ao (aorta) in the examined ferrets. In all cases a significant correlation was observed: LVWd and LVWs (a, b): $11.7 \pm 1.6 \mathrm{~mm}, \mathrm{p}<0.0001$ and $8.0 \pm 1.7 \mathrm{~mm}, \mathrm{p}=0.0003$, respectively; $\mathrm{LA}$ (c) and aorta (d) in M-mode: $7.2 \pm 1.3, \mathrm{p}=0.0077$ and $5.9 \pm 0.6 \mathrm{~mm}, \mathrm{p}<0.0001$, respectively; LA (e) and aorta (f) in B-mode: $6.7 \pm 1.2 \mathrm{~mm}$ and $5.3 \pm 0.6 \mathrm{~mm}$, both $\mathrm{p}<0.0001$. Source: $\odot \mathrm{N}$. Hildebrandt.

schied zwischen weiblichen und männlichen Tieren, wobei Letztere ein signifikant höheres Gewicht hatten. Die Betrachtung des Gewichts und nicht eines geschlechtsspezifischen Richtwerts erscheint sinnvoll, da sich nach Ergebnissen der vorliegenden Studie ( $>\mathbf{A b b} . \mathbf{3}$ ) und anderer Untersuchungen [11][12] das Gewicht einiger männlicher Frettchen nicht von dem der weiblichen Tiere abhob. Einschränkend ist zu bemerken, dass sich das Gewicht von Frettchen in Abhängigkeit von Jahreszeit und Sexualstatus ändert [20].

Die linksventrikulären Durchmesser ergaben in der aktuellen Studie in Diastole (LVDd 11,7 $\pm 1,6 \mathrm{~mm}$ ) wie auch in Systole (LVDs $8,0 \pm 1,7 \mathrm{~mm}$ ) vergleichbare Werte, wie sie in einer früheren Studie am wachen Frettchen (Median ca. 11,7 mm bzw. 8,0 mm) ermittelt worden waren [12]. Diese Werte liegen höher als bei Tieren, die mit Ketamin/Midazolam (Median 8,8 mm bzw. 5,9 mm) sediert [10] oder mit Isofluran ( $9,6 \mathrm{~mm}$ bzw. $6,9 \mathrm{~mm})$ in Narkose gehalten wurden [11]. Mögliche Erklärungen hierfür sind die unterschiedlichen Gewichtsbereiche in den Studien sowie eine Veränderung der Hämodynamik durch die Sedation/Narkose (z. B. Herzfrequenzsteigerung nach Ketaminapplikation, Reduktion des totalen peripheren Widerstands/systemischen Blutdrucks unter Isoflurannarkose), wie sie sowohl für das Frettchen als auch für andere Tierarten wie Hund oder Katze beschrieben sind [21][22] [23][24]. Insgesamt bestätigt die aktuelle Untersuchung, dass in der klinischen Routine spezifische Referenzwerte für wache Frettchen verwendet werden sollten.

Das linke Atrium und die Aorta konnten in der vorliegenden Studie bei allen Tieren sowohl im M-Mode-Verfahren als auch im zweidimensionalen Bild erfasst werden. Die Problematik der feh- lenden Mehrfachmessung im B-Bild wurde oben bereits diskutiert. Die absoluten Dimensionen von LA und Ao im M-Mode decken sich mit den Resultaten andere Studien an Frettchen mit ähnlichem Gewichtsbereich [10][12]. Der in der aktuellen Studie erstmals für das Frettchen gezeigte Einfluss des Gewichts auf die Größendimension von Aorta und Vorhof ist für andere Tierarten bekannt [19][25] und macht die Auswertung eines LA/Ao-Quotienten sinnvoll, da dieser unabhängig vom Gewicht ist. Das in der vorliegenden Studie ermittelte LA/Ao-Verhältnis im M-Mode $(1,2 \pm 0,1)$ deckt sich mit den Werten anderer Arbeiten, sowohl beim sedierten $(1,3 \pm 0,3)$ als auch beim narkotisierten (1,3 $\pm 0,2)$ Frettchen [10][11]. Die Messung im B-Bild im Vergleich zum M-Mode ergab in der vorliegenden Arbeit keinen signifikanten Unterschied. Die Angaben für gesunde Hunde [14] entsprechen dieser Aussage (B-Mode: 1,03 \pm 0,09, Range 0,84-1,27; M-Mode: 1,01 $\pm 0,13$, Range 0,71-1,41), während bei gesunden Katzen [15] die mit den beiden Messmethoden ermittelten Werte differierten (B-Mode: Median 1,40, Range 1,141,60; M-Mode: Median 1,44; Range 1,21-1,79).

Die Ermittlung des LA/Ao-Verhältnisses im B-Bild der kurzen Achse wird insbesondere beim Hund zur Erkennung einer Vorhofvergrößerung im Frühstadium einer degenerativen Mitralklappenerkrankung verwendet [13]. Inwieweit bei Frettchen mit Kardiopathien das B-Bild mit Messung des Vorhofkörpers besser geeignet ist als die Messung des Übergangs des Vorhofs zum Herzohr im M-Mode, muss in klinischen Studien untersucht werden. Eine entsprechende Erfassung sollte daher weiterführend bei Tieren mit unterschiedlicher Vorhofvergrößerung angestrebt werden, um die verschiedenen Messverfahren genauer bewerten zu können. 


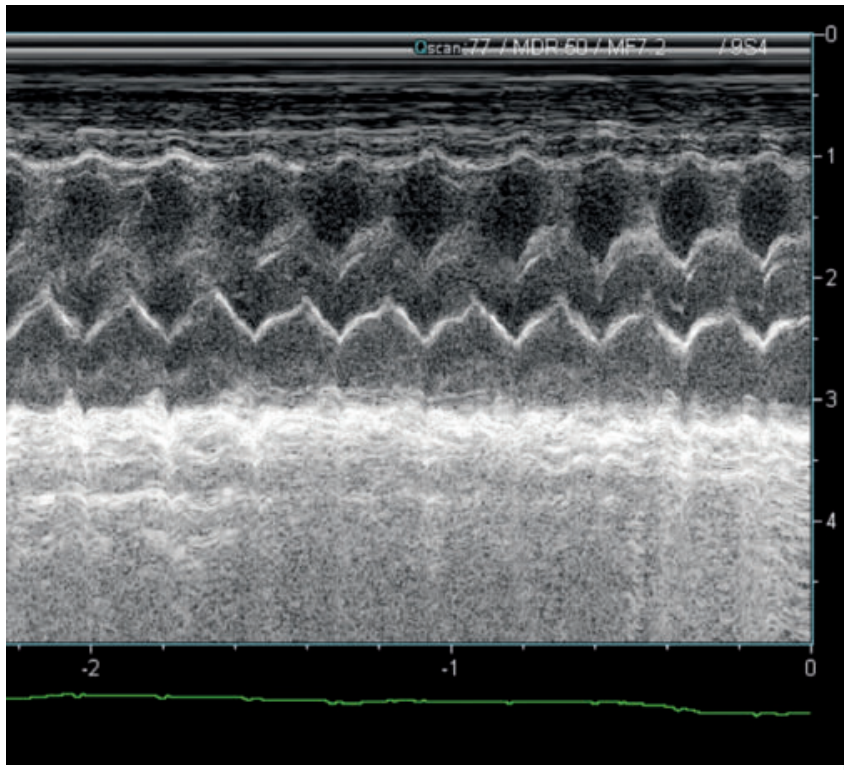

Abb. 5 M-Mode-Darstellung aus der rechtsparasternalen Längsachse mit Blick in den linksventrikulären Ausflusstrakt senkrecht durch die Aortenwurzel. Aufgrund suboptimaler Anschallung durch Bewegung des Tieres kann nur am Ende dieser Aufzeichnung eine eindeutige Messung der Aorta und des linken Vorhofs vorgenommen werden und eine Mehrfachmessung ist in diesem Fall nicht möglicht. Quelle: @ N. Hildebrandt.

-Fig.5 M-mode image from the right parasternal long axis view at the level of the aortic root and left atrium. Due to movement of the animal a correct clear measurement of aorta and left atrium can only be performed at the end of this image and therefore multiple measurements in that particular case are impossible. Source: (c) N. Hildebrandt.

\section{FAZIT FÜR DIE PRAXIS}

Beim gesunden Frettchen besteht eine Gewichstabhängigkeit von echokardiografischen Größendimensionen. Das Verhältnis von linkem Vorhof zu Aorta kann aus M-Mode und B-Bild erfasst werden. Der Quotient unterscheidet sich bei gesunden Tieren nicht und ist gewichtsunabhängig.

Interessenkonflikt

Die Autoren bestätigen, dass kein Interessenkonflikt besteht.

\section{Literatur}

[1] Di Girolamo N, Critelli M, Zeyen U et al. Ventricular septal defect in a ferret (Mustela putorius furo). J Small Anim Pract 2012; 53: 549-533

[2] Dias S, Planellas M, Canturri A et al. Extreme Tetralogy of Fallot with polycythemia in a ferret (Mustela putorius furo). Topics in Compan Anim Med 2017; 32: 80-85

[3] Wagner RA. Ferret cardiology. Vet Clin North Am Exot Anim Pract 2009; 12: 115-134
[4] Atkinson RM. Case reports on cardiomyopathy in the domestic ferret, Mustela putorius furo. J Small Exot Anim Med 1992; 2: 75-78

[5] Hildebrandt N, Schneider M. Die dilatative Kardiomyopathie beim Frettchen (Mustela putorius furo) - Symptomatik, Diagnostik und therapeutische Möglichkeiten. Tierärztl Prax 2009; 37 (K): 115-123

[6] Malakoff RL, Laste NJ, Orcutt C]. Echocardiographic and electrocardiographic findings in client-owned ferrets: 95 cases (1994-2009). J Am Vet Med Assoc 2012; 241: 1484-1489

[7] Sasai H, Kato K, Sasaki T et al. Echocardiographic diagnosis of dirofilariasis in a ferret. J Small Anim Pract 2000; 41: 172-174

[8] Sanchez-Migallon Guzman D, Mayer J, Melidone R et al. Pacemaker implantation in a ferret (Mustela putorius furo) with third-degree atrioventricular block. Vet Clin Exot Anim 2006; 9: 677-687

[9] Williams LA. Congestive heart failure and other medical facts about ferrets. Int J Pharm Compd 2009; 13: 367-369

[10] Stepien RL, Benson KG, Wenholz LJ. M-mode and Doppler echocardiographic findings in normal ferrets sedated with ketamine hydrochlorid and midazolam. Vet Radiol Ultrasound 2000; 41: 452-456

[11] Vastenburg MAHC, Boroffka SAEB, Schoemaker NJ. Echocardiographic measurements in clinicaly healthy ferrets anesthetized with isoflurane. Vet Radiol Ultrasound 2004; 45: 228-232

[12] Dudas-Györki Z, Szabo Z, Manczur F et al. Echocardiographic and electrocardiographic examination of clinically healthy, conscious ferrets. J Small Anim Pract 2011; 52: 18-25

[13] Boswood A, Häggström J, Gordon SG et al. Effect of pimobendan in dogs with preclinical myxomatous mitral valve disease and cardiomegaly: The EPIC study - a randomized clinical trial. J Vet Intern Med 2016; 30: 1765-1779

[14] Hansson K, Häggström J, Kvart C et al. Left atrial to aortic root indicies using two dimensional and M-mode echocardiography in Cavalier King Charles Spaniels with and without left atrial enlargement. Vet Radiol Ultrasound 2002; 43: 569-575

[15] Maerz I, Schober K, Oechtering G. Echokardiographische Untersuchung der linksatrialen Größe bei gesunden Katzen und mit linksventrikulärer Hypertrophie. Tierärztl Prax 2006; 34 (K): 331-340

[16] Thomas WP, Gaber CE, Jacobs G] et al. Recommenadations for standards in transthoracic two-dimensional echocardiography in the dog and cat. J Vet Intern Med 1993; 7: 247-252

[17] Sahn DJ, DeMaria A, Kisslo j et al. Recommendations regarding quantitation in M-mode echocardiography: results of a survey of echocardiographic measurements. Circulation 1978; 58 (6): 1072-1083

[18] Cornell CC, Kittleson MD, Torre PD et al. Allometric scalling of m-mode cardiac measurements in normal adult dogs. J Vet Intern Med 2004; 18: $311-321$

[19] Schober K, Savino S, Yildiz V. Reference intervals and allometric scaling of two-dimensional echocardiographic measurements in 150 healthy cats. J Vet Med Sci 2017; 79: 1764-1771

[20] Rosenthal K. Ferrets. Vet Clin North Am Small Anim Pract 1994; 24 $1-23$

[21] Imai A, Steffey EP, Farver TB et al. Assessment of isoflurane-induced anesthesia in ferrets and rats. Am J Vet Res 1999; 60: 1577-1583

[22] Jacobs G, Knight DH. Change in M-mode echocardiographic values in cats given ketamine. Am J Vet Res 1985; 46: 1712-1713

[23] Klide AM. Cardiopulmonary effects of enflurane and isoflurane in the dog. Am J Vet Res 1976; 37: 127-131

[24] Moreland AF, Glaser C. Evaluation of ketamine, ketamine-xylazine and ketamin-diazepam anesthesia in the ferret. Lab Anim Sci 1985; 35: 287-290

[25] Rishniw M, Caivano D, Dickson D et al. Two-dimensional echocardiographic left-atrial-to-aortic-ratio in healthy adult dogs: a reexamination of reference intervals. J Vet Cardiol 2019; 26: 29-38 